\title{
Seleção de extratos brutos de plantas com atividade antiobesidade
}

SOUZA, S.P. ${ }^{* *}$; PEREIRA, L.L.S. ${ }^{2}$; SOUZA, A.A. ${ }^{3}$; SANTOS, C.D. ${ }^{2}$

${ }^{1}$ Departamento de Química, Instituto Militar de Engenharia, Urca, CEP: 22290-270, Rio de Janeiro-Brasil *stefaniasouza@far.fiocruz.br 2Departamento de Química, Universidade Federal de Lavras, Caixa Postal 3037, CEP: 37200-000 Lavras-Brasil ${ }^{3}$ Departamento de Nutrição, Universidade Federal de Alfenas, Caixa Postal 37, CEP: 130-000, Alfenas-Brasil

RESUMO: A prevalência da obesidade está aumentando a um ritmo alarmante e poucos medicamentos para o tratamento estão atualmente disponíveis no mercado. A inibição da lipase pancreática é um dos mecanismos mais amplamente estudados para determinar a eficácia de produtos naturais como agentes antiobesidade. O objetivo do estudo foi avaliar in vitro a inibição de dezenove extratos aquosos e metanólicos sobre a lipase pancreática. Ensaios cinéticos foram realizados em quatro períodos de tempo com ausência e presença do extrato inibidor. Também foi avaliado a ação desses extratos após a simulação do fluido gástrico. Cymbopogon citratus apresentou a maior inibição, seguido de Costus spicatus e Baccharis trimera. Os extratos apresentam um potencial como adjuvante no tratamento da obesidade.

Palavras-chave: anti obesidade, extratos vegetais, inibição de lipídeos

ABSTRACT: Selection of crude plant extracts with anti-obesity activity. The prevalence of obesity has been increasing at an alarming rate and only a few medications are currently available in the market. Pancreatic lipase inhibition is one of the most widely studied mechanisms for determining the efficacy of natural products as anti-obesity agents. The aim of this study was to evaluate in vitro the inhibition of nineteen aqueous and methanol extracts on pancreatic lipase. Kinetic assays were performed in four periods with the absence and the presence of the inhibitor extract. We also tested the action of these extracts after gastric fluid simulation. Cymbopogon citratus showed the highest inhibition, followed by Costus spicatus and Baccharis trimera. The extracts have the potential to act as adjuvants in the treatment of obesity.

Key words: anti-obesity, plant extracts, lipid inhibition

\section{INTRODUÇÃO}

O metabolismo lipídico é mantido equilibrado para manter a homeostase. Quando o equilíbrio é perdido, desenvolve-se um quadro de obesidade ou de hiperlipidemia, levando a uma variedade de doenças graves, incluindo aterosclerose, hipertensão, diabetes e depressão funcional em determinados órgãos (Oliveira et al., 2004). A obesidade já é considerada epidemia mundial, independente de condições econômicas e sociais. O Brasil ocupa o 6o lugar no ranking mundial de países com problemas com obesidade. Os gastos diretos com esta doença, o que inclui internações, consultas e medicamentos, chegam a 1,1 bilhão de reais por ano, que equivale a $12 \%$ do total de gastos anuais do Sistema Único de Saúde (SUS) com internações (Gigante et al., 2009).

Portanto, o controle do metabolismo lipídico por drogas é uma alternativa que pode ser utilizada para prevenir ou tratar a obesidade. Um número crescente de enzimas envolvidas nas vias metabólicas lipídicas está sendo identificado e representa um conjunto rico de alvos terapêuticos potenciais para a obesidade e outros distúrbios metabólicos (Oliveira et al., 2001). Uma das estratégias mais importantes no tratamento da obesidade inclui o desenvolvimento de inibidores da digestão e absorção de nutrientes, em uma tentativa de reduzir a ingestão de energia por meio de mecanismos gastrintestinais, sem alterar qualquer mecanismo do sistema nervoso central. Os lipídios da dieta representam a maior fonte de calorias indesejadas e são compostos de, aproximadamente, $90 \%$ de triglicerídios. A enzima lipase pancreática é responsável pela hidrólise, para posterior absorção (Almeida et al., 2009).

Recebido para publicação em 17/10/2010

Aceito para publicação em 28/11/2011

Rev. Bras. PI. Med., Botucatu, v.14, n.4, p.643-648, 2012. 
Uma nova abordagem para tratamentos de redução de peso é inibir a digestão e a absorção de triglicerídios (inibindo a lipase pancreática). Fitoquímicos identificados a partir de plantas medicinais tradicionais representam excelente oportunidade para o desenvolvimento de terapêuticas antiobesidade (Birari \& Bhutani, 2007).

No Brasil, o Ministério da Saúde regulamentou, em 2006, a proposta de Política Nacional de Práticas Integrativas e Complementares no SUS (PNPIC-SUS), tendo como objetivos estabelecer a relação nacional de medicamentos fitoterápicos para atenção básica; incentivar a pesquisa e o desenvolvimento de medicamentos fitoterápicos a partir de plantas medicinais e resgatar, valorizar, embasar cientificamente e validar o conhecimento, a produção e o uso popular de plantas medicinais. O objetivo desta política é garantir à população brasileira o acesso seguro e o uso racional de plantas medicinal e fitoterápico, promovendo a utilização sustentável da biodiversidade e o desenvolvimento da cadeia produtiva e da indústria nacional (Brasil, 2006).

A etnofarmacologia vem ao encontro desses objetivos, uma vez que tem como finalidade básica, em estudos fitoquímicos e farmacológicos, conciliar as informações adquiridas junto às comunidades locais que fazem uso da flora medicinal, no intuito de fornecer dados para a pesquisa de medicamentos economicamente acessíveis à população mundial, especialmente de países em desenvolvimento.

Nesse contexto, plantas que são popularmente conhecidas como auxiliares na perda de peso tornam-se alvo central de pesquisas que visam o desenvolvimento de fitoterápicos que possam auxiliar no tratamento antiobesidade.

\section{MATERIALE MÉTODO}

\section{Seleção das plantas medicinais}

Foram selecionadas plantas medicinais com indicação de uso tradicional como agentes redutores de peso, hipocolesterolêmicas, espasmódica e laxativa. Nas Tabelas 1 e 2 estão relacionadas as plantas selecionadas para o estudo.

\section{Obtenção do material vegetal}

O material vegetal fresco das espécies foi obtido da coleção do Horto de Plantas Medicinais da Universidade Federal de Lavras (Tabela 1). As demais amostras foram obtidas no comércio local na forma desidratada, constituindo drogas vegetais pulverizadas (Tabela 2). As informações etnobotânicas foram obtidas no Herbário ESAL, do Departamento de Biologia da UFLA.

\section{Preparo do extrato aquoso a frio}

O material vegetal fresco (Tabela 1) foi lavado, fracionado em pedaços de aproximadamente $1 \mathrm{~cm}^{2}$ e triturado em gral de porcelana. Em seguida, foi preparado o extrato aquoso por maceração dinâmica na proporção 1:10 (p/v). Utilizou-se agitador horizontal por 30 minutos, em banho de gelo. Em seguida, a amostra foi centrifugada, a $1.700 \times$ g, por 10 minutos, a $4^{\circ} \mathrm{C}$. O sobrenadante foi recolhido e o extrato aquoso obtido foi utilizado nos ensaios de inibição da enzima lipase pancreática.

TABELA 1. Identificação do material vegetal obtido no Horto Medicinal e a parte da planta utilizada*.

\begin{tabular}{lll}
\hline Nome científico & Família & Parte utilizada \\
\hline Arctium lappa L. & Asteraceae & Folhas \\
Baccharis trimera (Less.) DC. & Asteraceae & Partes aéreas \\
Cassia angustifólia Vahl. & Fabaceae & Folhas \\
Cassia fistula L. & Fabaceae & Folhas \\
Coffea arabica L. & Rubiaceae & Folhas \\
Cordia salicifolia Cham. & Boraginaceae & Partes aéreas \\
Costus spicatus (Jacq.) S.W. & Zingiberaceas & Folhas \\
Cymbopogon citratus (DC.) Stapf & Poaceae & Folhas \\
Cynara cardunculus subsp. scolymus & Asteraceae & Partes aéreas \\
Echinodorus grandiflorus (Cham. \& Schltdl.) Micheli. & Alismatáceas & Folhas \\
Equisetum arvense L. & Equisetaceae & Partes aéreas \\
Medicago sativa L. & Fabaceae & Partes aéreas \\
Petroselinum crispum (Mill.) Nym. & Apiaceae & Partes aéreas \\
Plectranthus barbatus (Andr.) & Monimiaceae & Folhas \\
\hline
\end{tabular}

*material vegetal empregado na forma fresca.

Rev. Bras. PI. Med., Botucatu, v.14, n.4, p.643-648, 2012. 
TABELA 2. Identificação do material vegetal obtido em comércio local e a parte da planta utilizada*.

\begin{tabular}{llll}
\hline Nome científico & Nome popular & Família & Parte utilizada \\
\hline Fucus vesiculosus L. & Fucus & Fucaceae & Talo \\
Malus communis Poir. & Maçã & Rosaceae & Cascas do fruto \\
Polymnia sonchifolia Poepp. & Yacon & Fabaceae & Tubérculos \\
Prosopis juliflora (Sw.) DC. & Algaroba & Fabaceae & Folhas \\
Humulus lupulus L. & Lúpulo & Cannabaceae & Inteira \\
\hline
\end{tabular}

*material vegetal empregado na forma desidratada.

O material vegetal seco (Tabela 2) foi pesado, adicionou-se água na proporção de 1:10 (p/v) e preparou-se o extrato aquoso por maceração dinâmica, nas mesmas condições já descritas.

\section{Preparo do extrato metanólico}

O material vegetal fresco foi lavado e fragmentado em pedaços de, aproximadamente, 1 $\mathrm{cm}^{2}$. Em seguida, foi preparado um extrato metanólico por maceração estática, durante 24 horas. $O$ extrato foi filtrado em malha de náilon de 100ìm e deixado em banho-maria, $a 5^{\circ} \mathrm{C}$, até a completa evaporação do solvente. Após a evaporação do solvente, foi adicionada água ao resíduo, na proporção 1:10 (p/v). Esse extrato foi centrifugado, a $1.700 \times \mathrm{g}$, por 10 minutos e o sobrenadante utilizado como inibidor nas análises de atividade enzimática (Kwon et al., 2003). O material vegetal seco foi pesado e preparou-se o extrato metanólico na proporção de 1:10 (p/v), nas mesmas condições já descritas (Souza et al., 2011; Souza et al., 2012).

Ensaio de atividade da lipase pancreática A enzima lipase pancreática suína (tipo II, SIGMA), na concentração de $10 \mathrm{~g} \mathrm{~L}^{-1}$, foi preparada

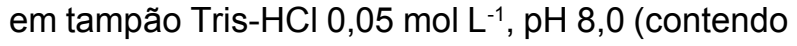
$\mathrm{CaCl}_{2}$ 0,010 $\left.\mathrm{mol} \mathrm{L}^{-1} \mathrm{e} \mathrm{NaCl} 0,025 \mathrm{~mol} \mathrm{~L}^{-1}\right)$. Como substrato utilizou-se o p-nitrofenilpalmitato na concentração de $0,008 \mathrm{~mol} \mathrm{~L}^{-1}$, dissolvido em Triton$X 100,0,5 \%(p / v)$.

Todas as análises foram feitas em triplicata. Em cada análise, a mistura de reação $(100 \mu \mathrm{L}$ de enzima, $50 \mu \mathrm{L}$ de água e $50 \mu \mathrm{L}$ de substrato) foi incubada por quatro períodos de tempo $(10,20,30 \mathrm{e}$ $40 \mathrm{~min}$.). Controles sem enzima (branco substrato) e sem substrato (branco enzima) foram incubados do mesmo modo que os tubos experimentais. A absorbância do produto da ação da lipase sobre o pnitrofenolpalmitato ( $p$-nitrofenol) foi determinada em espectrofotômetro no $\lambda$ de $410 \mathrm{~nm}$. A inclinação da reta do gráfico, Absorbância x Tempo, foi calculada e utilizada como referência para o cálculo da inibição da lipase pancreática (Souza et al., 2011; Souza et al., 2012).
Ensaio de inibição da lipase pancreática Os extratos vegetais aquosos a frio e metanólico, preparados conforme os itens 4.3 e 4.4 , foram submetidos a ensaios de inibição da enzima lipase pancreática. A inibição foi obtida a partir da inclinação da reta do gráfico Absorbância x Tempo. A atividade da lipase foi determinada da seguinte maneira:

(A): na ausência do extrato (possível inibidor), que corresponde ao ensaio enzima controle,

(a): na ausência do extrato e da enzima (branco substrato) e substrato

(B): na presença do extrato com a enzima + substrato).

(b): na ausência da enzima (branco extrato

Em cada análise, os extratos foram préincubados com a enzima por 10 minutos antes da adição do substrato. A porcentagem de inibição (I) foi quantificada pela equação:

$$
I \%=\frac{(A-a)-(B-b)}{(A-a)} \times 100
$$

A atividade da enzima inibida foi calculada a partir da diferença entre as inclinações na ausência do inibidor (A-a) e na presença do inibidor (B-b). Os resultados foram expressos em atividade de enzima inibida por grama de planta ( $\mathrm{ALI} \mathrm{g}^{-1}$ ) que corresponde a $1 \mu \mathrm{mol}$ de $\mathrm{p}$-nitrofenol que deixou de ser produzido por minuto, devido à presença do inibidor nas condições de ensaio (Souza et al., 2011; Souza et al., 2012).

\section{Simulação da ação do fluido gástrico}

Os extratos que apresentaram inibição significativa sobre a enzima lipase pancreática foram incubados com o fluido gástrico simulado preparado segundo a USP (United States Pharmacopeia, 1995), por 1 hora, em banho-maria, a $37^{\circ} \mathrm{C}$. Após esse período, foram neutralizados com bicarbonato de sódio puro até pH 8 e a inibição da enzima novamente determinada. 


\section{RESULTADO E DISCUSSÃO}

Dos extratos aquosos testados, nenhum apresentou atividade inibitória sobre a lipase pancreática. Observou-se ativação da enzima nas avaliações com os extratos aquosos. A ativação da lipase decorre da estabilização da superfície não polar do sítio ativo, através do contato deste com um ambiente apolar. Essa ativação tem sido descrita por alguns autores e a ação ativadora relacionada as saponinas presentes em grandes concentrações nas plantas (Maggio, 1956; Kato \& Tosa, 1983; Jahromi \& Ray, 1993; Nagem et al., 1994; Nagem et al., 1995; Lima et al., 2004).

A atividade de inibição dos extratos metanólicos, ressuspendidos em água, estão apresentadas na Tabela 3. O resultado expresso em porcentagem de inibição é relativo à atividade da enzima nas condições do ensaio, por isso os resultados foram transformados em atividade de lipase inibida por grama de planta $\left(A L I g^{-1}\right)$, um resultado absoluto. Cymbopogon citratus (355 $\mathrm{ALI} \mathrm{g}^{-1}$ ) apresentou a maior inibição, seguido de Costus spicatus (249 ALI $\left.\mathrm{g}^{-1}\right)$ e Baccharis trimera (241 ALI g-1 $)$.

A IV Diretriz da Sociedade Brasileira de Cardiologia (SBC, 2007) afirma que plantas com alto teor de flavonóides são utilizadas na prevenção e tratamento de hiperlipidemia, hipercolesterolemia e aterosclerose. A presença de flavonóides nas plantas que apresentaram atividade de inibição no presente estudo, descrita por outros pesquisadores, Baccharis trimera (Soicke e Leng-Peschlow, 1987; Bianchi et al., 1993; Gené et al., 1996; Gianello et al., 2000; Sharp et al., 2001), Costus spicatus (Silva et al., 2000), Cymbopogon citratus (Olaniyi et al., 1975; Sousa et al., 1991; Figueirinha et al., 2008), Cynara cardunculus (Noldin et al., 2003, Bezakova et al., 2007), Equisetum arvense (Bredebach et al., 2011; Gallo et al., 2011; Kolomiets et al., 2012), Echinodorus grandiflorus (Duarte et al., 2002), Malus communis (Matsubara \& Rodriguez-Amaya, 2006; Prasanna et al., 2012) sugerem que esses metabólitos secundários sejam responsáveis por essa inibição.

Os extratos que inibiram a enzima em maior porcentagem foram submetidos a um teste de simulação da ação do fluido gástrico sobre a atividade inibitória dos extratos (Tabela 4). $O$ extrato de $C$. arabica, após a simulação, perdeu a atividade, o que indica que o inibidor presente não é resistente à passagem pelo fluido gástrico. Tal resultado pode esclarecer a ausência de efeito hipolipidêmico, constatado por Souza et al. (2010). A inibição da lipase pelos extratos de $C$. citratus, $C$. cardunculus e $B$. trimera diminuiu, enquanto que a dos extratos de $C$. spicatus e E. grandiflorus foi mantida após a simulação do fluido gástrico. A exposição das moléculas ao fluido gástrico pode acarretar

TABELA 3. Inibição da lipase pancreática na presença dos extratos metanolicos.

\begin{tabular}{llcc}
\hline № & Nome científico & \%inibição** & Atividade de lipase inibida (ALI g $\left.^{-1}\right)^{* *}$ \\
\hline 1 & Arctium lappa L. & 0 & 0 \\
2 & Baccharis trimera (Less.) DC. & $78 \pm 0,06$ & $241 \pm 0,1$ \\
3 & Cassia angustifolia Vahl. & $*$ & 0 \\
4 & Cassia fistula L. & $*$ & 0 \\
5 & Coffea arabica L. & $53,2 \pm 0,2$ & $145 \pm 0,4$ \\
6 & Cordia salicifolia Cham. & $*$ & 0 \\
7 & Costus spicatus (Jacq.) S.W. & $40 \pm 0,1$ & $249 \pm 0,2$ \\
8 & Cymbopogon citratus (DC.) Stapf & $82 \pm 0,1$ & $355 \pm 0,1$ \\
9 & Cynara cardunculus subsp. scolymus & $68,6 \pm 0,2$ & $142 \pm 0,09$ \\
10 & Echinodorus grandiflorus (Cham. \& Schltdl.) Micheli. & $85,4 \pm 0,3$ & $176 \pm 0,6$ \\
11 & Equisetum arvense L. & $17 \pm 0,14$ & $131,5 \pm 0,3$ \\
12 & Fucus vesiculosus L. & $*$ & 0 \\
13 & Humulus lupulus & 0 & 0 \\
14 & Malus communis Poir. & $24,1 \pm 0,08$ & $114,7 \pm 0,1$ \\
15 & Medicago sativa L. & $*$ & 0 \\
16 & Petroselinum crispum (Mill.) Nym. & 0 & 0 \\
17 & Peumus boldus & 0 & 0 \\
18 & Polymnia sonchifolia Poepp. & $*$ & 0 \\
19 & Prosopis juliflora (Sw.) DC. & $*$ & 0 \\
\hline
\end{tabular}

*extratos que aumentaram a atividade da enzima; ${ }^{* *}$ média de três repetições \pm desvio padrão. 
TABELA 4. Atividade da lipase pancreática na presença de extratos metanólicos após simulação da digestão.

\begin{tabular}{|c|c|c|}
\hline Planta & $\begin{array}{l}\text { \% inibição após } \\
\text { simulação* }\end{array}$ & $\begin{array}{l}\text { Atividade de lipase inibida } \\
\qquad\left(A L I g^{-1}\right)^{*}\end{array}$ \\
\hline Baccharis trimera & $51 \pm 0,05$ & $225 \pm 0,1$ \\
\hline Coffea arabica & 0 & 0 \\
\hline Costus spicatus & $40 \pm 0,2$ & $247 \pm 0,14$ \\
\hline Cymbopogon citratus & $65 \pm 0,3$ & $297 \pm 0,4$ \\
\hline Cynara cardunculus & $40 \pm 0,07$ & $88 \pm 0,2$ \\
\hline Echinodorus grandiflorus & $84 \pm 0,08$ & $175 \pm 0,18$ \\
\hline
\end{tabular}

* média de 3 repetições \pm desvio padrão

modificações na estrutura do inibidor em decorrência do $\mathrm{pH}$ ácido do estômago ou da presença das proteinases, diminuindo ou inativando-os.

Agbafor \& Akubugwo (2007) investigaram a ação hipocolesterolêmica do extrato etanólico de $C$. citratus em ratos albinos e observaram que a concentração sérica de colesterol foi reduzida nos animais tratados com o extrato. Souza et al. (2012) avaliaram o efeito do extrato metanólico de carqueja em ratos com obesidade induzida por dieta rica em gorduras e observaram redução no peso e nos níveis de colesterol sérico dos animais. Resultados que corroboram com a inibição da lipase pancreática verificada no presente estudo.

Os resultados de inibição encontrados para essas plantas estão relatados pela primeira vez neste estudo. A manutenção da inibição após o fluido gástrico simulado sugere que o resultado tende a se repetir em ensaios in vivo. A inibição da lipase verificada sugere que Baccharis trimera, Costus spicatus, Cymbopogon citratus e Echinodorus grandiflorus podem ser promissoras no auxílio do tratamento da obesidade e da hipercolesterolemia.

\section{CONCLUSÃO}

Inibição da lipase pancreática é um dos mecanismos mais amplamente estudados para determinar o potencial de produtos naturais como agentes antiobesidade. Os extratos metanólicos de capim-limão (Cymbopogon citratus), alcachofra (Cynara cardunculus), cana-do-brejo (Costus spicatus), chapéu-de-couro (Echinodorus grandiflorus) e carqueja (Baccharis trimera) apresentam potencial como adjuvante no tratamento da obesidade e de dislipidemias, uma vez que inibem a atividade da enzima lipase pancreática.

\section{REFERÊNCIA}

AGBAFOR, K.N.; AKUBUGWO, E.L. Hypocholesterolaemic effect of ethanolic extract of fresh leaves of Cymbopogon citrates (lemongrass). African Journal of Biotechnology v.6, n.5, p.596-8, 2007.

ALMEIDA, J.C. et al. Papel dos lipídeos da dieta na nefropatia diabética. Arquivos Brasileiros Endocrinologia Metabolismo, v.53, n.5, p.634-45, 2009. BEZAKOVA, L. et al. Effect of flavonoids and cynarine from Cynara cardunculus L. on lipoxygenase activity. Acta Facultatis Pharmaceuticae Universitatis Comenianae, v.54, p.48-53, 2007.

BIANCHI, N.R. et al. Ensaio de toxicidade excessiva e screening fitoquímico de algumas espécies do gênero Baccharis L. (Asteraceae). Revista Brasileira de Farmácia, v.74, n.3, p.79-80, 1993.

BIRARI, R.B.; BHUTANI, K.K. Pancreatic lipase inhibitors from natural sources: unexplored potential. Drug Discovery Today, v.12, n.19/20, p.879-89, 2007.

BRASIL. Ministério da Saúde. Secretaria de Atenção à Saúde. Departamento de Atenção Básica. Política Nacional de Práticas Integrativas e Complementares no SUS - PNPIC-SUS. Brasília, 2006. 92p.

BREDEBACH, M.; MATERN, U.; MARTENS, S. Three 2oxoglutarate-dependent dioxygenase activities of Equisetumarvense $\mathrm{L}$. forming flavone and flavonol from (2S)-naringenin. Phytochemistry, v.72, n.7, p.557-63, 2011. DUARTE, M.G.R. et al. Perfil fitoquímico e atividade antibacteriana in vitro de plantas invasoras. Revista Lecta, v.20, n.2, p.177-82, 2002.

FIGUEIRINHA, A. et al. Cymbopogon citratus leaves: Characterization of flavonoids by HPLC-PDA-ESI/MS/ MS and an approach to their potential as a source of bioactive polyphenols. Food Chemistry, v.110, n.3, p.71828, 2008.

GALLO, F.R. et al. Chemical fingerprinting of Equisetum arvense L. using HPTLC densitometry and HPLC. Natural Product Research: Formerly Natural Product Letters, v.25, n.13, p.1261-70, 2011.

GENÉ, R.M. et al. Anti-inflammatory and analgesic activity of Baccharis trimera: identification of its active constituents. Planta Medica, v.62, p.232-5, 1996.

GIANELLO, J.C. et al. Medicamentos herbários en el centro-oeste argentino. II. "Carquejas": control de calidad de lãs drogas oficiales y sustituyentes. Acta Farmaceutica Bonaerense, v.19, n.2, p.99-103, 2000.

GIGANTE, D.P.; MOURA, E.C.; SARDINHA, L.M.V. Prevalência de excesso de peso e obesidade e fatores associados, Brasil, 2006. Revista Saúde Pública, v.43, n.2, p.83-89, 2009.

JAHROMI, M.A.F.; RAY, A.B. Antiherlipidemic effect of 
flavonoids from Pterocarpus marsupium. Journal of Natural Products, v.56, n.7, p.989-94, 1993.

KATO, N.; TOSA, N. Effects of dietary quercetin on serum lipids. Agricultural Biological Chemistry, v.47, n.9, p.2119-20, 1983.

KOLOMIETS, N.E.; YUSUBOV, M.S.; KALINKINA, G.I. Flavonoid composition of Equisetum arvense and $E$. litorale studied by high-performance liquid chromatography-mass spectrometry. Chemistry of Natural Compounds, v.48, n.1, p.135-6, 2012

KWON, C.S. et al. Anti-obesity effect of Dioscorea nipponica Makino with lipase inhibitory activity in rodents. Bioscience, Biotechnology, and Biochemistry, v.67, n.7, p.1451-6, 2003.

LIMA, L.R.P. et al. Influência de produtos naturais na ativação da lipase pancreática humana. Revista Ciências Farmacêuticas, v.25, n.1, p.25-9, 2004.

MAGGIO, G.D. Azione della quercetina sull andamento di alcuni processi patologici sperimentali corretali alla mallatia arteriosclerotica. Rivista Italiano de Science Famacologche, p.28-37, 1956.

MATOUSCHEK, B.V.; STAHL-BISKUP, E. Phytochemische untersuchung der nichflüchtigeninhaltsstoffe von Cymbopogon citratus (DC.) Stapf (Poaceae). Pharmaceutica Acta Helvetiae, v.66, s.p., 1991. MATSUBARA, S.; RODRIGUEZ-AMAYA, D.B. Conteúdo de miricetina, quercetina e kaempferol em chás comercializados no Brasil . Ciência Tecnologia Alimentos, v.26, n.2, p.380-5, 2006.

NAGEM,T.J. et al. Efeito de flavonóides sobre lipídeos em ratos e sobre enzimas metabolizadoras de drogas. Arquivos de Biologia e Tecnologia, v.37, n.3, p.471-82, 1994.

NAGEM, T.J. et al. Efeitos de derivados flavonoídicos sobre lipídeos em ratos. Arquivos de Biologia e Tecnologia, v.38, n.3, p.859-68, 1995.

NOLDIN, V.F. et al. Composição química e atividades biológicas das folhas de Cynara scolymus L. (alcachofra) cultivada no Brasil. Química Nova, v.26, n.3, p.1-9, 2003. OLANIYI, A.A.; SOFOWORA, E.A.; OGUNTIMEHIN, B.O. Phytochemical investigation of some Nigerian plants used against fevers. II. Cymbopogon citratus. Planta Medica, v.28, p.186-9, 1975.
OLIVEIRA, T.T. et al. Efeito hipolidêmico e sinérgico da naringina, clorofila e monascus em ratos (Ratus novergicus). Alimentos e Nutrição, v.12, n.1, p.95-102, 2001.

OLIVEIRA, C.L. et al. Obesidade e síndrome metabólica na infância e adolescência. Revista de Nutrição, v.17, n.2, p.237-45, 2004.

PRASANNA, T.V. et al. In vitro antioxidant activities of few seasonal fruits. Pharmaceutical Research, v.2, n.2, p.28-30, 2012.

SHARP, $\mathrm{H}$. et al. 6-Oxygenated flavones from Baccharis trinervis (Asteraceae). Biochemical Systematics and Ecology, v.29, p.105-7, 2001.

SILVA, B.P.; BERNARDO, R.R.; PARENTE, J.P. Flavonol glycosides from Costus spicatus. Phytochemistry, v.53, p.87-92, 2000.

SOCIEDADE BRASILEIRA DE CARDIOLOGIA-SBC. IV Diretriz Brasileira Sobre Dislipidemias e Diretriz de Prevenção da Aterosclerose do Departamento de Aterosclerose da Sociedade Brasileira de Cardiologia. Arquivos Brasileiros de Cardiologia, v.88, supl. 1, p.119, 2007.

SOICKE, H.; LENG-PESCHLOW, E. Characterization of flavonoids from Baccharis trimera and their antihepatotoxic properties. Planta Medica, v.53, p.37-9, 1987.

SOUSA, M.P. et al. Constituintes químicos ativos de plantas medicinais brasileiras. Fortaleza: Editora da UFC, 1991. 448p.

SOUZA, R.M.N.et al. Teores de compostos bioativos em cafés torrados e moídos comerciais. Quimica Nova, v.33, n.4, p.885-90, 2010.

SOUZA, S.P. et al. Inhibition of pancreatic lipase by extracts of Baccharis trimera (Less.) DC., Asteraceae: evaluation of antinutrients and effect on glycosidases. Brazilian Journal of Pharmacognosy, v.13, n.1, p.12-8, 2011.

SOUZA, S.P. et al. Estudo da atividade antiobesidade do extrato metanólico de Baccharis trimera (Less.) DC. Brazilian Journal of Pharmacy, v.93, n.1, p.27-32, 2012. The United States Pharmacopeia-USP. The National Formulary NF 18 (Pharmacopeial Convention Inc.) Rockville, MD.Janeiro. 1995. 